\title{
Research Review: Neural response to threat in children, adolescents, and adults after child maltreatment - a quantitative meta-analysis
}

\author{
Tyler C. Hein and Christopher S. Monk \\ Department of Psychology, University of Michigan, Ann Arbor, MI, USA
}

\begin{abstract}
Background: Child maltreatment is common and has long-term consequences for affective function. Investigations of neural consequences of maltreatment have focused on the amygdala. However, developmental neuroscience indicates that other brain regions are also likely to be affected by child maltreatment, particularly in the social information processing network (SIPN). We conducted a quantitative meta-analysis to: confirm that maltreatment is related to greater bilateral amygdala activation in a large sample that was pooled across studies; investigate other SIPN structures that are likely candidates for altered function; and conduct a data-driven examination to identify additional regions that show altered activation in maltreated children, teens, and adults. Methods: We conducted an activation likelihood estimation analysis with 1,733 participants across 20 studies of emotion processing in maltreated individuals. Results: Maltreatment is associated with increased bilateral amygdala activation to emotional faces. One SIPN structure is altered: superior temporal gyrus, of the detection node, is hyperactive in maltreated individuals. The results of the whole-brain corrected analysis also show hyperactivation of the parahippocampal gyrus and insula in maltreated individuals. Conclusions: The meta-analysis confirms that maltreatment is related to increased bilateral amygdala reactivity and also shows that maltreatment affects multiple additional structures in the brain that have received little attention in the literature. Thus, although the majority of studies examining maltreatment and brain function have focused on the amygdala, these findings indicate that the neural consequences of child maltreatment involve a broader network of structures. Keywords: Maltreatment; meta-analysis; fMRI.
\end{abstract}

\section{Introduction}

Incidence estimates for child maltreatment are as high as 1 in 4 (CDC, 2015). Maltreatment encompasses both abuse and neglect, and refers to injury, death, emotional harm, or serious risk of harm to a child due to a caregiver's actions or failure to act (Childhelp, n.d.). Child maltreatment is linked to significant risk of injury as well as to many negative health and behavior outcomes in adolescence and adulthood (Dubowitz \& Poole, 2012; Hussey, Chang, $\&$ Kotch, 2006). These consequences, which include mental health, contribute to the high human and societal cost (Buckingham \& Daniolos, 2013; Wang $\&$ Holton, 2007). One study estimates that the total lifetime cost of maltreatment is $\$ 124$ billion annually (Fang, Brown, Florence, \& Mercy, 2012). Thus, it is crucial to gain a better understanding of the effects of maltreatment. A likely mediator is the brain; child maltreatment may alter brain development in a way that changes socioemotional function and increases the likelihood that an individual will develop a mental disorder. By aggregating the findings of neuroimaging investigations of child maltreatment, we sought to characterize how this early experience impacts neural function.

Child maltreatment typically occurs in a social context. Young children depend on caregivers for

Conflict of interest statement: No conflicts declared. love and safety. When caregivers deviate from this expected social relationship, brain development within neural structures that are involved in social processing may be impacted. Therefore, brain regions associated with emotional facial expression processing are likely to be involved in the connection between child maltreatment and later psychopathology. The social information processing network (SIPN) (Nelson, Leibenluft, McClure, \& Pine, 2005) describes specific brain regions that may be affected by maltreatment. The SIPN is divided into three nodes: a detection node, an affective node, and a cognitive-regulatory node. The detection node is responsible for identifying whether a stimulus is social in nature, and includes inferior occipital cortex, inferior temporal cortex, intraparietal sulcus, fusiform face area, superior temporal sulcus, and anterior temporal cortex. The affective node further processes the social and emotional components of a stimulus, and includes the amygdala, ventral striatum, septum, bed nucleus of the stria terminalis, hypothalamus, and orbitofrontal cortex. Finally, the cognitive-regulatory node engages in complex processes such as response inhibition or goal-directed behavior, and includes the dorsomedial prefrontal cortex, as well as the dorsal and ventral prefrontal cortices. Many of these brain regions, particularly neocortical structures and the amygdala, experience significant postnatal development, making them differentially susceptible to the effects of 
maltreatment (Teicher et al., 2003). Given that brain development during childhood is extensive, it is reasonable to expect that the long-term functional consequences of early-life stress extend across many areas of the brain. Additionally, the brain undergoes significant structural and functional changes between childhood and adulthood; it is possible that youth and adults who experienced child maltreatment may differ in their neural reactivity.

Neuroimaging research highlights brain regions involved in the relationship between child maltreatment and socioemotional deficits. The amygdala is frequently implicated. Furthermore, altered amygdala function is linked to disorders that have increased prevalence in child maltreatment survivors, such as depression and posttraumatic stress disorder (PTSD) (Buckingham \& Daniolos, 2013). Neuroimaging consistently documents amygdala hyperactivity in response to emotional stimuli in those who experienced child maltreatment (Bogdan, Williamson, \& Hariri, 2012; De Bellis \& Hooper, 2012; Maheu et al., 2010; McCrory et al., 2011; Suzuki et al., 2014; Tottenham et al., 2011).

Other regions involved in socioemotional processing are also affected by early-life stress. Previously institutionalized children show increased activation in dorsal prefrontal cortex, subgenual anterior cingulate, temporal cortex, parietal cortex, and cerebellum (Tottenham et al., 2011). Adolescents exposed to early-life stress evidence abnormal activity in the anterior cingulate cortex, prefrontal cortex, striatum, and insula (Mueller et al., 2010). In summary, child maltreatment contributes to alterations in limbic and frontal areas; two regions that are involved in socioemotional processing and undergo protracted development.

Despite the premise that maltreatment broadly impacts the brain, most studies examine selective regions of interest (ROIs). While this reduces type 1 error, it limits what can be learned about the consequences of child maltreatment. The most common ROI in child maltreatment research is the amygdala (Bogdan et al., 2012; Dannlowski et al., 2013; Fonzo et al., 2013; Herringa, Phillips, Fournier, Kronhaus, \& Germain, 2013; Maheu et al., 2010; McCrory et al., 2011; Nicol, Pope, Romaniuk, \& Hall, 2015; Redlich et al., 2015; Tottenham et al., 2011; van Harmelen et al., 2013; White et al., 2012). Other frontal or limbic brain regions are rarely selected as ROIs. When a whole-brain approach is used, findings outside of the amygdala are seldom discussed in detail. In order to elucidate the mechanisms involved in the relationship between child maltreatment and psychopathology, it is important to gain understanding of how this early-life stressor affects the entire brain.

We conducted a quantitative meta-analysis of neuroimaging studies comparing neural response to emotional stimuli in maltreated and control individuals to determine which brain regions are impacted by child maltreatment. By conducting a meta-analysis, which requires synthesis of multiple datasets into a single brain map, we avoid inconsistencies in brain region labeling across studies, which can interfere with narrative-based reviews (Kühn \& Gallinat, 2013), and can determine statistically significant patterns of activation. Furthermore, the large sample size from combining datasets resulted in increased statistical power. We conducted this meta-analysis to facilitate understanding of overall trends across neuroimaging studies of child maltreatment. Activation likelihood estimation (ALE) is a well-established method for conducting a quantitative meta-analysis. ALE is a coordinate-based technique that uses peak activation coordinates and participant number to model voxelwise convergence in activation foci (Laird et al., 2005; Turkeltaub, Eden, Jones, \& Zeffiro, 2002). Benefits of ALE include utilization of coordinates instead of regions, as well as usage of a null distribution to enable statistical testing (Chase, Eickhoff, Laird, \& Hogarth, 2011). The results of ALE analyses indicate regions that have increased activation over all included studies, corrected for the entire brain. Our primary analyses aggregated all studies to attain maximum sample size and power.

Our goals for the meta-analysis were: (a) Evaluate whether maltreatment is related to greater bilateral amygdala activation; (b) Examine links of maltreatment with other social information processing network structures; (c) Conduct a data-driven examination of regions that experience altered activation in maltreated individuals; (d) Compare neural consequences of child maltreatment in youth and adults. We hypothesized the following: (a) maltreatment would relate to increased bilateral amygdala activation; (b) maltreatment would be associated with altered activation of other SIPN regions; (c) the data-driven examination would identify additional structures that are also linked to maltreatment; (d) maltreated youth and adults would both demonstrate increased bilateral amygdala activation.

\section{Methods Study selection}

A literature search of neuroimaging studies comparing patterns of activation in individuals with early-life stress to controls was conducted in March 2016. Our search terms were as follows: [(maltreat* OR abuse* OR advers* OR stress* OR famil* OR poverty*) AND (amygdala* OR cortic* OR brain* OR fMRI* OR imaging OR activ*)]. The initial search yielded approximately 10,900 results in Google Scholar. The literature search was repeated using PsycINFO and Pubmed. After a review of potential candidates for the analysis, we realized that there was a wide range of tasks used in these neuroimaging studies of individuals exposed to early maltreatment. The majority of tasks, however, involved processing of emotional faces stimuli, which is essential to successful socioemotional function. In order to isolate brain activation associated with socioemotional function, we decided to only include tasks that used emotional faces as stimuli. As maltreatment likely 
involves exposure to fear, anger, and sadness, we decided to limit the analysis to papers that focused on contrasting al faces, fear, anger, neutral, or sad faces with a neutral, scrambled, or baseline condition. These decisions narrowed our potential candidates down to 136 articles.

Abstracts of all identified articles were screened for the following criteria: were studies of individuals exposed to child maltreatment; utilized tasks that probed emotional facial expression processing; used fMRI; used image subtraction to produce activation foci; reported activation foci as 3-D coordinates; provided Talairach or MNI coordinates. About 78 articles were eliminated after this stage of screening (Figure 1). The 58 remaining articles were read in full in order to screen for the criteria. Another 38 articles were eliminated, leaving 20 studies for the analysis (Table 1). All studies utilize tasks that involve processing of emotional faces and include participants that have been exposed to abuse or neglect.

These studies yielded a total of 149 foci, or XYZ coordinates of activation, for individuals with a history of maltreatment and 26 foci for control participants. For analytic purposes, any foci, or activation clusters, not reported according to the atlas of the Montreal Neurological Institute (MNI coordinates) were converted to MNI coordinates using the icbm2tal transformation (Laird et al., 2010; Lancaster et al., 2007). For papers that only presented ROI results, the corresponding author was asked for whole-brain results.

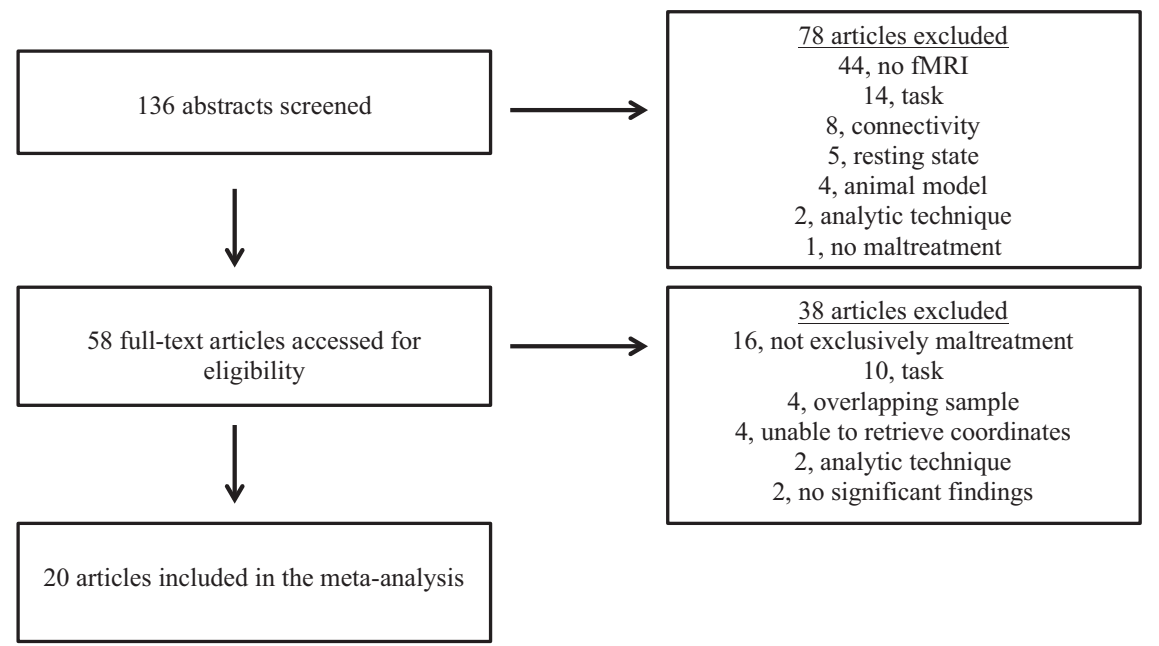

Figure 1 Flowchart of literature search

Table 1 Studies included in the meta-analysis

\begin{tabular}{|c|c|c|c|c|}
\hline Paper & Age & $N$ & Risk & Emotional faces task \\
\hline Bogdan et al. (2012) & Youth (11-15 years) & 279 & Emotional Neglect (CTQ) & Hariri (EFAT) \\
\hline $\begin{array}{l}\text { Crozier, Wang, Huettel, \& De } \\
\text { Bellis (2014) }\end{array}$ & Youth (11-13 years) & 74 & Abuse, Neglect (CPS confirmed) & Oddball \\
\hline Dannlowski et al. (2013) & Adults & 150 & Child Maltreatment (CTQ) & $\begin{array}{l}\text { Subliminal Affective } \\
\text { Priming }\end{array}$ \\
\hline De Bellis \& Hooper (2012) & Youth (10-18 years) & 16 & Abuse, Neglect (CPS confirmed) & Oddball \\
\hline Fonzo et al. (2013) & Adults & 33 & Child Maltreatment (CTQ) & Hariri (EFAT) \\
\hline $\begin{array}{l}\text { Ganzel, Kim, Gilmore, } \\
\text { Tottenham, \& Temple (2013) }\end{array}$ & Youth(10-15 years) & 14 & Severe Life Events (CIDI) & Passive Viewing \\
\hline Garrett et al. (2012) & Youth (10-16) & 46 & Interpersonal Trauma & Gender Discrimination \\
\hline Gee et al. (2013) & Youth (6.5-17.6 years) & 89 & Previous Institutionalization & Indicate Neutral Faces \\
\hline Herringa et al. (2013) & Adults & 28 & Child Maltreatment (CTQ) & Dynamic Faces \\
\hline Jedd et al. (2015) & Adults & 71 & $\begin{array}{l}\text { Child Maltreatment (DHS } \\
\text { confirmed) }\end{array}$ & Hariri (EFAT) \\
\hline Lee et al. (2015) & Adults & 31 & Verbal Abuse (VAQ) & Gender Discrimination \\
\hline Maheu et al. (2010) & Youth (9-18 years) & 30 & Previous Institutionalization & Altering Attention \\
\hline McCrory et al. (2011) & Children & 43 & Exposure to Violence & Gender Discrimination \\
\hline $\begin{array}{l}\text { McLaughlin, Peverill, Gold, } \\
\text { Alves, \& Sheridan (2015) }\end{array}$ & Youth (13-19 years) & 42 & Child Maltreatment (CTQ) & Emotion Regulation \\
\hline Nicol et al. 2015 & Adults & 36 & Child Trauma (CTQ) & Gender Discrimination \\
\hline Redlich et al. (2015) & Adults & 377 & Child Maltreatment (CTQ) & Hariri (EFAT) \\
\hline $\begin{array}{l}\text { Skokauskas, Carballedo, } \\
\text { Fagan, \& Frodl (2015) }\end{array}$ & Adults & 80 & Sexual Abuse (CTQ) & Attention Shifting \\
\hline Suzuki et al. (2014) & Children & 115 & Early-Life Trauma & Gender Discrimination \\
\hline Tottenham et al. (2011) & Children & 44 & Previous Institutionalization & Go/No-Go \\
\hline van Harmelen et al. (2013) & Adults & 135 & $\begin{array}{l}\text { Child Emotional Maltreatment } \\
\text { (NEMESIS) }\end{array}$ & Gender Discrimination \\
\hline
\end{tabular}

Total $N=1,733$. CIDI $=$ Composite International Diagnostic Interview; CTQ $=$ Childhood Trauma Questionnaire; CPS $=$ Child Protective Services; DHS $=$ Department of Human Services; EFAT $=$ Emotional Face Assessment Task; VAQ $=$ Verbal Abuse Questionnaire. 


\section{Meta-analysis}

All meta-analyses were performed using ALE (Turkeltaub et al., 2002). We used GingerALE software (version 2.3.6) to conduct the meta-analysis (Eickhoff, Bdzok, Laird, Kurth, \& Fox, 2012; Eickhoff et al., 2009; Turkeltaub et al., 2012). Using the MNI peak coordinates reported by the studies listed in Table 1, we conducted the analyses. One analysis used foci reflecting greater activation in the maltreatment group, and the other used foci reflecting greater activation in the control group. For each group of foci, activation likelihood estimates were calculated for each voxel of the brain. Foci are modeled as the center of three-dimensional Gaussian distributions, which are summed across experiments to reflect interstudy consistencies in activation. The cluster-wise family-wise error (FWE) correction was employed to correct for multiple comparisons at a significance threshold of $p<.05$ (Eickhoff et al., 2016). ALE analyses use nonparametric analyses, and are therefore not susceptible to the inflated false-positive rates in cluster-wise analyses (Eklund, Nichols, \& Knutsson, 2016).

\section{Results}

\section{Objective 1: Amygdala}

Our meta-analysis indicated significantly increased activation in bilateral amygdala for individuals with maltreatment relative to controls (Table 2, Figure 2).

\section{Objective 2: SIPN regions}

The right superior temporal gyrus, a region of the SIPN detection node, was more active in individuals with maltreatment relative to controls (Table 2).

\section{Objective 3: Data-Driven Examination}

Outside of the social information processing network, a whole-brain corrected analysis revealed two brain regions had increased activation in maltreated individuals: bilateral parahippocampal gyrus and the right insula (Table 2).

\section{Control > maltreatment}

Results of the meta-analysis for control $>$ maltreatment found no effects.

\section{Analyses with whole-brain studies only}

The ideal input for an ALE meta-analysis is wholebrain data, but given the frequency with which ROI approaches are used, it can be impractical to omit all non-whole-brain data (Laird, 2008). Instead, it is helpful to include ROI data, but then run a second analysis with only whole-brain data to ensure that the findings still hold. Of the 20 studies in our metaanalysis, 18 included whole-brain findings in the original article $(N=1,660)$. The remaining two studies did not include whole-brain analyses; these studies focused on the amygdala (Maheu et al., 2010; McCrory et al., 2011), hippocampus (Maheu et al., 2010), and anterior insula (McCrory et al., 2011). The corresponding author for each of these studies was asked to provide the whole-brain output corresponding to the article. For a variety of reasons, it was not possible for any of the authors to fulfill the requests. To examine the influence of whole-brain reporting, the meta-analyses were rerun with wholebrain studies only.

The results of this were overall similar to the results of the analyses including all studies. One important difference was that increased activation in the left parahippocampal gyrus and amygdala became nonsignificant when the analyses were run with only whole-brain studies. Increased activation in the left lentiform nucleus and globus pallidus region in maltreated individuals became significant. Activation in the right parahippocampal gyrus and right amygdala remained. Other differences in findings between the two sets of analyses related to the size of implicated areas. In the analyses of wholebrain studies only, the size of right parahippocampal gyrus and right amygdala region was decreased. Furthermore, the size of the right insula and superior temporal gyrus region that was more likely to be activated in maltreated individuals was increased in the analyses using whole-brain studies only.

\section{Adult versus youth studies}

Our meta-analysis includes studies of both youth and adults who experienced childhood maltreatment. There were not enough articles to perform a direct statistical comparison using GingerALE, but we ran our analyses with youth $(N=11)$ and adult $(N=9)$ studies separately in order to qualitatively compare their results preliminarily.

Results of both the youth and adult analyses showed increased right parahippocampal and amygdala activation in maltreated individuals.

Table 2 Results of the ALE analysis comparing maltreated and control individuals

\begin{tabular}{|c|c|c|c|c|c|c|c|}
\hline Contrast & Cluster Number & Volume $\left(\mathrm{mm}^{3}\right)$ & Brain region & $X$ & Y & $Z$ & Extrema value \\
\hline \multirow[t]{4}{*}{ Maltx $>\mathrm{C}$} & 1 & 2856 & $\begin{array}{l}\text { L Parahippocampal Gyrus/Amygdala } \\
\text { L Parahippocampal Gyrus/Amygdala }\end{array}$ & $\begin{array}{l}-22 \\
-24\end{array}$ & $\begin{array}{r}-6 \\
0\end{array}$ & $\begin{array}{l}-14 \\
-20\end{array}$ & $\begin{array}{l}0.019769501 \\
0.019481266\end{array}$ \\
\hline & 2 & 2312 & R Parahippocampal Gyrus/Amygdala & 26 & 0 & -22 & 0.034059726 \\
\hline & 3 & 680 & R Insula/Brodmann Area 13 & 48 & -34 & 22 & 0.016861271 \\
\hline & & & R Superior Temporal Gyrus & 52 & -32 & 16 & 0.015429311 \\
\hline
\end{tabular}

Maltx = maltreated $\mathrm{C}=$ control; $\mathrm{L}=$ left $\mathrm{R}=$ right; $X, Y, Z$ coordinates refer to MNI space; cluster-wise FWE correction 


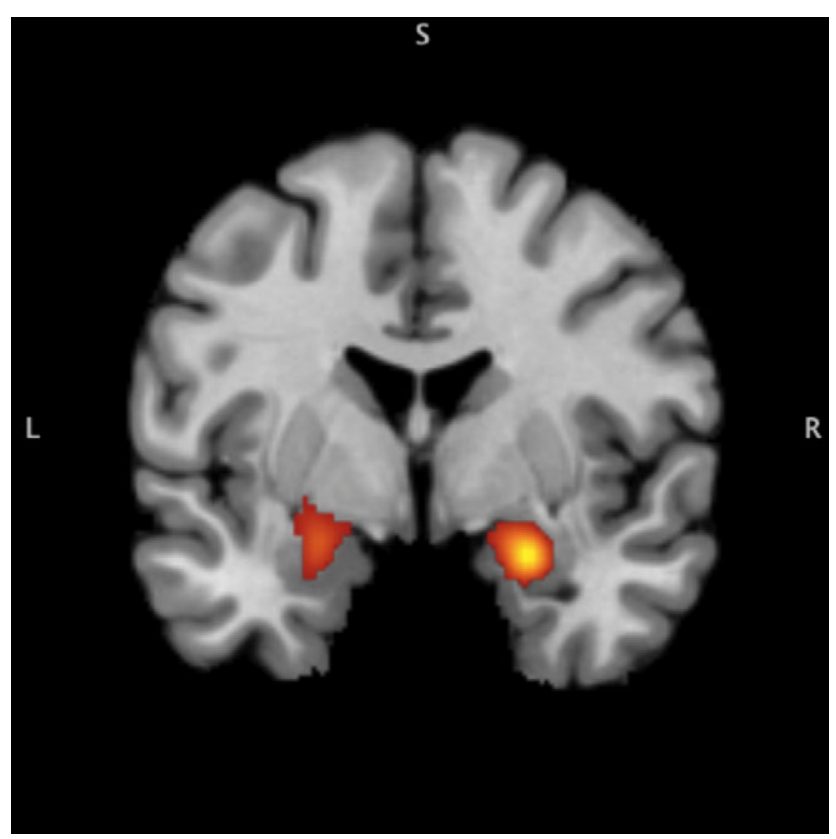

Figure 2 Bilateral amygdala and parahippocampal gyrus results from the activation likelihood estimation (ALE) analyses. Notes: Maltreated $>$ Control. Cluster-wise FWE $p<.05$. [Colour figure can be viewed at wileyonlinelibrary.com]

Additionally, maltreated youth, but not adults, demonstrated hyperactivation in the left lentiform nucleus and globus pallidus, as well as in the left parahippocampal gyrus.

\section{Discussion}

This was the first quantitative meta-analysis to examine the effects of child maltreatment on brain function. Relative to controls, the maltreated group exhibits increased bilateral amygdala activation. Additionally, maltreated individuals evidence increased activation in the superior temporal gyrus, a region in the SIPN detection node. Finally, two other regions outside of the SIPN, the parahippocampal gyrus and insula, show increased activation in the maltreated group.

As hypothesized, bilateral amygdala is hyperactive in individuals that have been maltreated. This is consistent with a review of child maltreatment neuroimaging literature that concluded the neural pathways most altered by child maltreatment are in fronto-limbic networks, including the amygdala (Hart \& Rubia, 2012). As this region is highly involved in emotional processes, including emotion regulation (Phelps \& LeDoux, 2005), altered amygdala activation may underlie the socioemotional difficulties associated with child maltreatment. Increased amygdala activation is linked to several forms of psychopathology, including depression (Siegle, Thompson, Carter, Steinhauer, \& Thase, 2007), anxiety (Phan, Fitzgerald, Nathan, \& Tancer, 2006), and PSTD (Shin, Rauch, \& Pitman, 2006). Increased amygdala activation may be adaptive during the experience of maltreatment, but increase risk of later psychopathology.
Beyond the amygdala, the superior temporal gyrus is hyperactive in maltreated individuals relative to controls. This brain region is linked to social perception and cognition (Pelphrey, Adolphs, \& Morris, 2004). Increased activation of the superior temporal gyrus may aid in early detection of emotion, which may enable a child to identify threatening emotion in their environment quickly, an adaptive ability in the context of child maltreatment. Social information flows from the detection node to the affective node to the cognitive-regulation node (Nelson et al., 2005). Our results indicate that maltreatment may lead to altered function at the detection and affective nodes. Abnormalities in emotion detection and affective response are likely to result in aberrant socioemotional function and an increased risk of psychopathology for some individuals.

Outside of the SIPN structures, the present metaanalysis also showed that hippocampal formation structures, bilateral parahippocampal gyri, are hyperactive in maltreated individuals in comparison to control individuals. Child maltreatment is associated with PTSD (Ackerman, Newton, McPherson, Jones, \& Dykman, 1998); altered hippocampal formation activation may be a risk factor that interacts with child maltreatment to produce PTSD, or a consequence of child maltreatment that predisposes an individual to later PTSD. The literature on functional alterations of the hippocampal formation in PTSD is mixed; parahippocampal activation tends to be increased and hippocampal activation has been found to be both decreased and increased (Francati, Vermetten, \& Bremner, 2007).

A second region outside the SIPN that our analysis identified is the insula, which is hyperactive in maltreated individuals. The insula has been linked to processing of subjective feelings and uncertainty, as well as empathy (Singer, Critchley, \& Preuschoff, 2009). Additionally, the anterior insula, which has been linked to integration of interoceptive and visceral information, is implicated in emotional experience by recent theoretical accounts (Eisenberger, 2015; Smith, Steinberg, \& Chein, 2014). This region is asserted to be involved in cognitiveemotional network interactions (Smith et al., 2014) and may be important for effective modulation of attention in the presence of emotional stimuli. Increased insula activation to emotional faces is linked to higher trait anxiety (Stein, Simmons, Feinstein, \& Paulus, 2007). Insula hyperactivation is also found in social anxiety disorder and specific phobia in a meta-analysis (Etkin \& Wager, 2007).

A third region that was identified only in the wholebrain and youth analyses consists of the lentiform nucleus and globus pallidus, which is hyperactive in maltreated individuals. These structures are located within the basal ganglia, which is implicated in the recognition of disgust (Adolphs, 2002) and motivation in a recent review (Ikemoto, Yang, \& Tan, 2015). Altered basal ganglia activity is linked to psychiatric 
disorders such as obsessive-compulsive disorder (OCD), anxiety, and depression (Gunaydin \& Kreitzer, 2016). The basal ganglia is connected to the prefrontal cortex (Alexander, Crutcher, \& DeLong, 1990); prefrontal cortical development during adolescence may result in levels of basal ganglia activation that are more similar to those of nonmaltreated individuals.

There were a number of limitations with this ALE meta-analysis. First, a relatively small number of studies were used in the analyses. Although ALE meta-analyses of this size are capable of producing results, increasing the number of studies could have produced a larger number of significant group differences (Fox, 2015). As more neuroimaging studies of individuals that were maltreated as children are published, it will be important to conduct additional meta-analyses. Nevertheless, 1,733 participants were included in this analysis, which provides a basis for more reliable findings and lays the groundwork for future studies to examine specific brain structures in relation to child maltreatment.

A second concern is the inability of several research groups to provide whole-brain coordinates for this meta-analysis, reducing our subsequent comparison analyses to 18 studies. Currently, there are many technical, financial, and social challenges to sharing fMRI data (Poldrack \& Gorgolewski, 2014). Without a consistent structure for storing fMRI data for future use, organization of these data falls to an individual or group of individuals, making identifying the location of these data years later difficult. In order to maximize the contribution of research participants and funding, it will be important to utilize effective ways of structuring data for sharing that investigators can implement at the time of data collection.

A third limitation is that we were not able to explore the effect of task in this meta-analysis. Although we tightly constrained our literature search to include tasks that used emotional faces, our final sample included studies with a range of emotionrelated tasks that are likely to use slightly different cognitive and neural resources. As neuroimaging studies of individuals who experienced childhood maltreatment increase in number, it will be important to evaluate the influence of this early experience on behavioral performance and neural response to specific emotion-related tasks.

A fourth limitation is that we were not able to parse apart variables, such as gender, age of exposure, and duration of maltreatment. It will be important to understand how these variables affect neural function at every step in development. It would be ideal to tightly constrain these variables in these neuroimaging studies. Nevertheless, given the difficulties of recruiting such individuals, it would be a significant challenge. Other variables that are important to consider are age of exposure and type of maltreatment. Responses to child maltreatment are likely to vary by age of exposure, as well as type of maltreatment; the neural response that physical abuse elicits in an infant is likely to differ from the neural response that emotional neglect elicits in an older child. As a result, the neural consequences of child maltreatment may vary by these factors. However, due to the realities of child maltreatment, specifically that is often chronic and involves multiple types of abuse or neglect, parsing apart the influence of these variables will be difficult. However, in a recent review, McLaughlin, Sheridan, and Lambert propose a differentiation between deprivation and threat that may be more feasible to implement in future analyses (McLaughlin, Sheridan, \& Lambert, 2014). Despite these limitations, the current meta-analysis provides the field with knowledge of the neural impacts of child maltreatment across multiple studies.

\section{Conclusions}

In a meta-analysis of 1,733 individuals, hyperactivation of the bilateral amygdala and parahippocampal gyri, right insula, and right superior temporal gyrus is related to child maltreatment. Going forward, we need longitudinal studies to better understand how maltreatment alters brain development. Specifically, such studies would allow investigators to identify the influence of important variables including age of exposure, type of maltreatment, duration of maltreatment, age at study participation, gender, and study task design. Meanwhile, this meta-analysis provides a more complete understanding of how early maltreatment impacts brain development. Findings from this work may be used in developing future hypotheses that involve structures beyond the amygdala and consider networks of brain structures.

\section{Acknowledgements}

This review was supported by the University of Michigan, Department of Psychology and the Horace $\mathrm{H}$. Rackham School of Graduate Studies. The authors thank Rebecca Waller for her contributions to this work. They declare they have no conflicts of interest in relation to this article.

\section{Correspondence}

Tyler C. Hein, 2010 East Hall, 530 Church St, Ann Arbor, MI 48109, USA; Email: heint@umich.edu 


\section{Key points}

- Maltreatment affects as many $25 \%$ of children and has long-term consequences for affective function.

- Most neuroimaging research of individuals with a history of child maltreatment has focused on the amygdala. However, it is unclear how maltreatment impacts other areas of the brain.

- We found that child maltreatment is related to bilateral amygdala reactivity and is also related to altered reactivity across several brain regions, including parahippocampal gyrus, insula, and superior temporal gyrus.

- These findings indicate that the neural consequences of child maltreatment extend beyond threat circuitry.

- Clinical research should consider the entire brain when investigating the neural consequences of child maltreatment.

\section{References}

*Indicates inclusion in the meta-analysis.

Ackerman, P.T., Newton, J.E., McPherson, W.B., Jones, J.G., \& Dykman, R.A. (1998). Prevalence of post traumatic stress disorder and other psychiatric diagnoses in three groups of abused children (sexual, physical, and both). Child Abuse and Neglect, 22, 759-774.

Adolphs, R. (2002). Neural systems for recognizing emotion. Current Opinion in Neurobiology, 12, 169-177.

Alexander, G.E., Crutcher, M.D., \& DeLong, M.R. (1990). Basal ganglia-thalamocortical circuits: Parallel substrates for motor, oculomotor", prefrontal" and "limbic" functions. Progress in Brain Research, 85, 119-146.

*Bogdan, R., Williamson, D.E., \& Hariri, A.R. (2012). Mineralocorticoid receptor iso/val (rs5522) genotype moderates the association between previous childhood emotional neglect and amygdala reactivity. American Journal of Psychiatry, 169, 515-522.

Buckingham, E.T., \& Daniolos, P. (2013). Longitudinal outcomes for victims of child abuse. Current Psychiatry Reports, 15, 1-7.

Chase, H.W., Eickhoff, S.B., Laird, A.R., \& Hogarth, L. (2011). The neural basis of drug stimulus processing and craving: An activation likelihood estimation meta-analysis. Biological Psychiatry, 70, 785-793.

Childhelp. (n.d.). The Issue of child abuse. Available from: https://www.childhelp.org/child-abuse [last accessed 6 October 2016].

Child Maltreatment Prevention. (2015, July 24). Available from: http://www.cdc.gov/violenceprevention/childmal treatment/ [last accessed 1 December 2015].

${ }^{*}$ Crozier, J.C., Wang, L., Huettel, S.A., \& De Bellis, M.D. (2014). Neural correlates of cognitive and affective processing in maltreated youth with posttraumatic stress symptoms: Does gender matter? Development and Psychopathology, 26, 491-513.

*Dannlowski, U., Kugel, H., Huber, F., Stuhrmann, A., Redlich, R., Grotegerd, D., ...\& \& Arolt, V. (2013). Childhood maltreatment is associated with an automatic negative emotion processing bias in the amygdala. Human Brain Mapping, 34, 2899-2909.

*De Bellis, M.D., \& Hooper, S.R. (2012). Neural substrates for processing task-irrelevant emotional distracters in maltreated adolescents with depressive disorders: A pilot study. Journal of Traumatic Stress, 25, 198-202.

Dubowitz, H., \& Poole, G. (2012). Child neglect: An overview. MacMillan H, topic ed. In: R.E. Tremblay, M. Boivin, \& R.De.V. Peters (Eds.), Encyclopedia on early childhood development [online] (pp. 1-6). Montreal, QC: Centre of Excellence for Early Childhood Development and Strategic Knowledge Cluster on Early Child Development. Available from: http://www.childencyclopedia.com/documents/DubowitzPooleANGxp1.pdf [last accessed 21 July 2015].
Eickhoff, S.B., Bdzok, D., Laird, A.R., Kurth, F., \& Fox, P.T. (2012). Activation likelihood estimation revisited. NeuroImage, 59, 2349-2361.

Eickhoff, S.B., Laird, A.R., Grefkes, C., Wang, L.E., Zilles, K., \& Fox, P.T. (2009). Coordinate-based activation likelihood estimation meta-analysis of neuroimaging data: A randomeffects approach based on empirical estimates of spatial uncertainty. Human Brain Mapping, 30, 2907-2926.

Eickhoff, S.B., Nichols, T.E., Laird, A.R., Hoffstaedter, F., Amunts, K., Fox, P.T., .. \& \& Eickhoff, C.R. (2016). Behavior, sensitivity, and power of activation likelihood estimation characterized by massive empirical simulation. NeuroImage, $137,70-85$.

Eisenberger, N.I. (2015). Social pain and the brain: Controversies, questions, and where to go from here. Annual Review of Psychology, 66, 601-629.

Eklund, A., Nichols, T.E., \& Knutsson, H. (2016). Cluster failure: Why fMRI inferences for spatial extent have inflated false-positive rates. Proceedings of the National Academy of Sciences, 113, 7900-7905.

Etkin, A., \& Wager, T.D. (2007). Functional neuroimaging of anxiety: A meta-analysis of emotional processing in PTSD, social anxiety disorder, and specific phobia. American Journal of Psychiatry, 164, 1476-1488.

Fang, X., Brown, D.S., Florence, C.S., \& Mercy, J.A. (2012). The economic burden of child maltreatment in the United States and implications for prevention. Child Abuse and Neglect, 36, 156-165.

*Fonzo, G.A., Flagan, T.M., Sullivan, S., Allard, C.B., Grimes, E.M., Simmons, A.N., ... \& Stein, M.B. (2013). Neural functional and structural correlates of childhood maltreatment in women with intimate-partner violence-related posttraumatic stress disorder. Psychiatry Research: Neuroimaging, 211, 93-103.

Fox, M. (2015, March 13). number of studies/foci [Msg 2]. Available from: http://www.brainmap.org/forum/view topic. php? $\mathrm{f}=3 \& \mathrm{t}=487 \& \mathrm{sid}=2 \mathrm{dd} 624 \mathrm{~b} 175 \mathrm{~d} 49 \mathrm{f} 6 \mathrm{~b} 366 \mathrm{e} 00 \mathrm{bd0} 4$ 9f353b [last accessed 9 October 2015].

Francati, V., Vermetten, E., \& Bremner, J.D. (2007). Functional neuroimaging studies in posttraumatic stress disorder: Review of current methods and findings. Depression and Anxiety, 24, 202-218.

*Ganzel, B.L., Kim, P., Gilmore, H., Tottenham, N., \& Temple, E. (2013). Stress and the healthy adolescent brain: Evidence for the neural embedding of life events. Development and Psychopathology, 25(4pt1), 879-889.

*Garrett, A.S., Carrión, V., Kletter, H., Karchemskiy, A., Weems, C.F., \& Reiss, A. (2012). Brain activation to facial expressions in youth with PTSD symptoms. Depression and Anxiety, 29, 449-459.

*Gee, D.G., Gabard-Durnam, L.J., Flannery, J., Goff, B., Humphreys, K.L., Telzer, E.H., ... \& Tottenham, N. (2013). Early developmental emergence of human amygdala-prefrontal connectivity after maternal deprivation. 
Proceedings of the National Academy of Sciences, 110, 15638-15643.

Gunaydin, L.A., \& Kreitzer, A.C. (2016). Cortico-basal ganglia circuit function in psychiatric disease. Annual Review of Physiology, 78, 327-350.

Hart, H., \& Rubia, K. (2012). Neuroimaging of child abuse: A critical review. Frontiers in Human Neuroscience, 6, 52.

*Herringa, R.J., Phillips, M.L., Fournier, J.C., Kronhaus, D.M., \& Germain, A. (2013). Childhood and adult trauma both correlate with dorsal anterior cingulate activation to threat in combat veterans. Psychological Medicine, 43, 1533-1542.

Hussey, J.M., Chang, J.J., \& Kotch, J.B. (2006). Child maltreatment in the United States: Prevalence, risk factors, and adolescent health consequences. Pediatrics, 118, 933942 .

Ikemoto, S., Yang, C., \& Tan, A. (2015). Basal ganglia circuit loops, dopamine and motivation: A review and enquiry. Behavioural Brain Research, 290, 17-31.

*Jedd, K., Hunt, R.H., Cicchetti, D., Hunt, E., Cowell, R.A., Rogosch, F.A., ... \& Thomas, K.M. (2015). Long-term consequences of childhood maltreatment: Altered amygdala functional connectivity. Development and Psychopathology, 27(4pt2), 1577-1589.

Kühn, S., \& Gallinat, J. (2013). Gray matter correlates of posttraumatic stress disorder: A quantitative meta-analysis. Biological Psychiatry, 73, 70-74.

Laird, A. (2008, April 22). ROI based studies [Msg 2]. Available from: http:/ /www.brainmap.org/forum/viewtopic.php?f=3\&t= $64 \& \operatorname{sid}=2 d d 624 b 175 d 49 f 6 b 366 e 00 b d 049 f 353 b$ [last accessed 22 July 2015].

Laird, A.R., Fox, P.M., Price, C.J., Glahn, D.C., Uecker, A.M., Lancaster, J.L., ... \& Fox, P.T. (2005). ALE meta-analysis: Controlling the false discovery rate and performing statistical contrasts. Human Brain Mapping, 25, 155-164.

Laird, A.R., Robinson, J.L., McMillan, K.M., TordesillasGutiérrez, D., Moran, S.T., Gonzales, S.M., ... \& Lancaster, J.L. (2010). Comparison of the disparity between Talairach and MNI coordinates in functional neuroimaging data: Validation of the Lancaster transform. NeuroImage, 51, 677-683.

Lancaster, J.L., Tordesillas-Gutierrez, D., Martinez, M., Salinas, F., Evans, A., Zilles, K., .. \& \& Fox, P.T. (2007). Bias between MNI and Talairach coordinates analyzed using the ICBM-152 brain template. Human Brain Mapping, 28, 1194 1205.

${ }^{*}$ Lee, S.W., Yoo, J.H., Kim, K.W., Lee, J.S., Kim, D., Park, H., . . $\&$ Jeong, B. (2015). Aberrant function of frontoamygdala circuits in adolescents with previous verbal abuse experiences. Neuropsychologia, 79, 76-85.

*Maheu, F.S., Dozier, M., Guyer, A.E., Mandell, D., Peloso, E., Poeth, K., ... \& Ernst, M. (2010). A preliminary study of medial temporal lobe function in youths with a history of caregiver deprivation and emotional neglect. Cognitive, Affective, and Behavioral Neuroscience, 10, 34-49.

*McCrory, E.J., De Brito, S.A., Sebastian, C.L., Mechelli, A., Bird, G., Kelly, P.A., \& Viding, E. (2011). Heightened neural reactivity to threat in child victims of family violence. Current Biology, 21, R947-R948.

*McLaughlin, K.A., Peverill, M., Gold, A.L., Alves, S., \& Sheridan, M.A. (2015). Child maltreatment and neural systems underlying emotion regulation. Journal of the American Academy of Child and Adolescent Psychiatry, 54, 753-762.

McLaughlin, K.A., Sheridan, M.A., \& Lambert, H.K. (2014). Childhood adversity and neural development: Deprivation and threat as distinct dimensions of early experience. Neuroscience and Biobehavioral Reviews, 47, 578-591.

Mueller, S.C., Maheu, F.S., Dozier, M., Peloso, E., Mandell, D., Leibenluft, E., .. \& \& Ernst, M. (2010). Early-life stress is associated with impairment in cognitive control in adolescence: An fMRI study. Neuropsychologia, 48, 3037-3044.
Nelson, E.E., Leibenluft, E., McClure, E., \& Pine, D.S. (2005). The social re-orientation of adolescence: A neuroscience perspective on the process and its relation to psychopathology. Psychological Medicine, 35, 163-174.

*Nicol, K., Pope, M., Romaniuk, L., \& Hall, J. (2015). Childhood trauma, midbrain activation and psychotic symptoms in borderline personality disorder. Translational Psychiatry, 5, e559.

Pelphrey, K., Adolphs, R., \& Morris, J.P. (2004). Neuroanatomical substrates of social cognition dysfunction in autism. Mental Retardation and Developmental Disabilities Research Reviews, 10, 259-271.

Phan, K.L., Fitzgerald, D.A., Nathan, P.J., \& Tancer, M.E. (2006). Association between amygdala hyperactivity to harsh faces and severity of social anxiety in generalized social phobia. Biological Psychiatry, 59, 424-429.

Phelps, E.A., \& LeDoux, J.E. (2005). Contributions of the amygdala to emotion processing: From animal models to human behavior. Neuron, 48, 175-187.

Poldrack, R.A., \& Gorgolewski, K.J. (2014). Making big data open: Data sharing in neuroimaging. Nature Neuroscience, 17, 1510-1517.

*Redlich, R., Stacey, D., Opel, N., Grotegerd, D., Dohm, K., Kugel, H., .. \& \& Dannlowski, U. (2015). Evidence of an IFN- $\gamma$ by early life stress interaction in the regulation of amygdala reactivity to emotional stimuli. Psychoneuroendocrinology, 62, 166-173.

Shin, L.M., Rauch, S.L., \& Pitman, R.K. (2006). Amygdala, medial prefrontal cortex, and hippocampal function in PTSD. Annals of the New York Academy of Sciences, 1071, 67-79.

Siegle, G.J., Thompson, W., Carter, C.S., Steinhauer, S.R., \& Thase, M.E. (2007). Increased amygdala and decreased dorsolateral prefrontal BOLD responses in unipolar depression: Related and independent features. Biological Psychiatry, 61, 198-209.

Singer, T., Critchley, H.D., \& Preuschoff, K. (2009). A common role of insula in feelings, empathy and uncertainty. Trends in Cognitive Sciences, 13, 334-340.

*Skokauskas, N., Carballedo, A., Fagan, A., \& Frodl, T. (2015). The role of sexual abuse on functional neuroimaging markers associated with major depressive disorder. The World Journal of Biological Psychiatry, 16, 513-520.

Smith, A.R., Steinberg, L., \& Chein, J. (2014). The role of the anterior insula in adolescent decision making. Developmental Neuroscience, 36, 196-209.

Stein, M.B., Simmons, A.N., Feinstein, J.S., \& Paulus, M.P. (2007). Increased amygdala and insula activation during emotion processing in anxiety-prone subjects. American Journal of Psychiatry, 164, 318-327.

*Suzuki, H., Luby, J.L., Botteron, K.N., Dietrich, R., McAvoy, M.P., \& Barch, D.M. (2014). Early life stress and trauma and enhanced limbic activation to emotionally valenced faces in depressed and healthy children. Journal of the American Academy of Child and Adolescent Psychiatry, 53, 800-813.

Teicher, M.H., Andersen, S.L., Polcari, A., Anderson, C.M., Navalta, C.P., \& Kim, D.M. (2003). The neurobiological consequences of early stress and childhood maltreatment. Neuroscience and Biobehavioral Reviews, 27, 33-44.

*Tottenham, N., Hare, T.A., Milner, A., Gilhooly, T., Zevin, J.D., \& Casey, B.J. (2011). Elevated amygdala response to faces following early deprivation. Developmental Science, 14, 190204.

Turkeltaub, P.E., Eden, G.F., Jones, K.M., \& Zeffiro, T.A. (2002). Meta-analysis of the functional neuroanatomy of single-word reading: Method and validation. NeuroImage, 16, 765-780.

Turkeltaub, P.E., Eickhoff, S.B., Laird, A.R., Fox, M., Wiener, M., \& Fox, P. (2012). Minimizing within-experiment and within-group effects in activation likelihood estimation meta-analyses. Human Brain Mapping, 33, 1-13. 
*van Harmelen, A.L., van Tol, M.J., Demenescu, L.R., van der Wee, N.J., Veltman, D.J., Aleman, A., ... \& Elzinga, B.M. (2013). Enhanced amygdala reactivity to emotional faces in adults reporting childhood emotional maltreatment. Social Cognitive and Affective Neuroscience, 8, 362-369.

Wang, C.T., \& Holton, J. (2007). Total estimated cost of child abuse and neglect in the United States. Chicago: Prevent Child Abuse America.
White, M.G., Bogdan, R., Fisher, P.M., Muñoz, K.E., Williamson, D.E., \& Hariri, A.R. (2012). FKBP5 and emotional neglect interact to predict individual differences in amygdala reactivity: FKBP5, emotional neglect and amygdala reactivity. Genes, Brain and Behavior, 11, 869-878.

Accepted for publication: 22 August 2016

First published online: 25 October 2016 